\title{
INFLUÊNCIA AGUDA DO USO DE DIFERENTES PROTETORES BUCAIS NA CAPACIDADE AERÓBICA, POTÊNCIA MUSCULAR E METABÓLICA EM ATLETA DE BASQUETEBOL: UM ESTUDO PILOTO
}

\author{
Arthur Azevedo dos Santos ${ }^{1 *}$,Victor de Souza Maciel ${ }^{1}$, Victor Paes Dias Gonçalves ${ }^{1}$, Israel Lucas \\ Gomes da Silva Teles ${ }^{1}$, Anderson Pontes Morales ${ }^{23}$ \& Marlana Ribeiro Monteiro ${ }^{3}$
}

SANTOS, A.A.; MACIEL, V.S.; GONÇALVES, V.P.D.; TELES, I.L.G.S.; MORALES, A.P.; MONTEIRO, M.R. Influência aguda do uso de diferentes protetores bucais na capacidade aeróbica, potência muscular e metabólica em atleta de basquetebol: Um estudo piloto. Perspectivas Online: Biológicas \& Saúde, v. 8, n 28, p.15-23, 2018.

\section{RESUMO}

Introdução: O uso de protetores bucais (PB) na prática de esportes tem sido de enorme importância para a prevenção de traumas bucais. No entanto, atletas hesitam em usar PB por causa da redução no desempenho. Objetivo: Avaliar a influência aguda do uso de diferentes $P B$ na capacidade aeróbica, potência muscular e metabólica em atleta de basquetebol profissional. Método: Foi realizado um estudo randomizado cruzado: sem protetor bucal (Controle), protetor bucal universal (PBU) e protetor bucal personalizado (PBP). $\mathrm{O}$ atleta realizou 2 baterias de teste constituído por 30 saltos verticais. Os saltos verticais foram realizados antes (AN) e imediatamente após (IA) a execução do teste da capacidade aeróbica (YO-YO Intermittent Recovery). Foi analisada a concentração de lactato sanguíneo AN e IA a execução dos dois protocolos. Resultado: $\mathrm{O}$ presente estudo evidencia de forma descritiva que o uso do PBP pode impactar no aumento da distância máxima percorrida pelo atleta, que consequentemente pode ter elevado à concentração de lactato sanguíneo. Já a manutenção da potência muscular foi similar entre os ensaios experimentais. Conclusão: Estes achados fornecem evidências adicionais para treinadores que defendem o uso de PB personalizados, não só para proteção orofacial, mas também na indução de modificações positivas em determinados parâmetros específicos como a capacidade aeróbica e metabólica avaliados no atleta de basquetebol classificado como respirador nasal.

Palavras-chave: Protetor Bucal; Performance; Basquetebol. 


\begin{abstract}
Introduction: The use of mouthguards (MG) in sports has been of great importance for the prevention of oral traumas. However, athletes hesitate to wear mouthguards because of reduced performance. Objective: To evaluate the acute influence of the use of different mouthguards on cardiorespiratory fitness, muscle power and metabolic in professional basketball athletes. Method: A randomized crossover study was performed: without mouthguard (Control), universal mouthguard (UMG) and personalized mouthguard (PMG). The athlete performed 2 test batteries consisting of 30 vertical jumps. Vertical jumps were performed before (B) and immediately after (IA) the performance of the

YO-YO Intermittent Recovery test. The blood lactate concentration B and IA were analyzed in the execution of the two protocols. Results: The present study shows that the use of PMG had an impact on the increase in the distance, which consequently higher blood lactate levels. Already the muscle power and behavior of the data were similar between the experimental trials. Conclusion: These findings provide additional evidence for coaches advocating the use of PMG, not only for orofacial protection, but also for modifications in certain specific parameters such as aerobic capacity and metabolic of the basketball athlete classified as a nasal respirator.
\end{abstract}

Keywords: Mouthguard; Performance; Basketball.

\footnotetext{
1 Institutos Superiores de Ensino do CENSA -ISECENSA - - Aluno Voluntário de Iniciação Científica PROVIC - Rua Salvador Correa, 139, Centro, Campos dos Goytacazes, RJ, CEP: 28035-310, Brasil.

2 Pesquisador (a) do Laboratório de Fisiologia e Performance Motora - LAFIPEM/ISECENSA

3 Pesquisador Laboratório de Química e Biomoléculas - LAQUIBIO/ISECENSA

(*)e-mail: arthurazevedo1998@ hotmail.com

Data de recebimento: 12/11/2018 Aceito para publicação: 13/12/2018
}

Persp. online: biol. \& saúde, Campos dos Goytacazes, 28 (8), 15-23, 2018

seer.perspectivasonline.com.br 


\section{INTRODUÇÃO}

O aumento da popularidade dos esportes de contatos e o elevado nível de competitividade deu origem ao um número considerável de lesões orofaciais (Castaldi, 1986; Castaldi, 1988). De 1990 a 2003 nos E.U.A, houve uma média de 22.000 lesões dentárias anualmente (Stewart et al., 2009). Em 2006 segundo a "National Youth Sports Safety Foundation", durante a prática de esporte de contato, os atletas têm até $10 \%$ mais chance de sofrer uma lesão orofacial em uma temporada e $33 \%$ a $56 \%$ de probabilidade de sofrê-la no decorrer de sua carreira (NYSSF, 2006). Do mesmo modo, mais de cinco milhões de elementos dentários são perdidos anualmente com a prática esportiva (Frontera, 2008).

O uso de protetores bucais (PB) na prática de esportes tem sido de enorme importância para a prevenção de traumas bucais, reduzindo em até 80\% o risco (NYSSF, 2006). Os PB eram bem toscos em meados do século XIX e os atletas (boxeadores) usavam diversos tipos de materiais (algodão, lã, esponja e fita) como protetores. Em seguida, esses PB passaram a ser fabricados em três tipos: tipo I - pré-fabricados ou universais; tipo II - "ferve e morde" ou moldáveis pelo calor; e tipo III, customizados ou personalizados, os quais são confeccionados pelo Cirurgião-Dentista (CD) (Queiróz, 2013).

Muitos atletas, particularmente aqueles que competem nos esportes de contato físico, usam PB para proteção. No entanto, outros atletas hesitam em usar PB por causa da incerteza em relação a potenciais decrementos no desempenho (Gebauer et al., 2011). Entretanto, tem-se observado uma melhoria no desempenho dos atletas usuários desses dispositivos (Queiróz et al., 2013). Há evidências de que o reposicionamento da mandíbula com o uso PB pode melhorar a força muscular (Kerr, 1986) e a capacidade cardiorrespiratória (Queiróz et al., 2013) . Além disso, vários estudos odontológicos relataram aumento da força, equilíbrio, coordenação e desempenho como resultado da mudança em relação à articulação maxilomandibular com uso dos PB (Kaufman, 1980; Schwart \& Novich, 1980; Piero et al., 2015).

O estudo de Gebauer et al. (2011), avaliou o consumo de oxigênio em várias intensidades de exercício, realizando três testes experimentais em esteira, randomizados, com o uso de um PB personalizado, e sem PB. Os resultados observados no estudo não apresentaram impacto negativo sobre ventilação pulmonar, consumo de oxigênio ou frequência cardíaca durante o exercício de diferentes intensidades com a utilização do PB personalizado.

Em um estudo realizado por Queiróz et al. (2013), o PB personalizado, ou seja, o tipo III apresentou os melhores resultados para o atleta, melhorando as variáveis da capacidade aeróbica quando comparado ao não uso de PB. Desta forma, são necessárias novas pesquisas para investigar a influência do uso de protetor na performance dos atletas, principalmente utilizando testes na ambiência do atleta. As pesquisas que se dedicam a este objetivo ainda são escassas na literatura demonstrando a importância deste estudo.

Diante desse cenário o presente estudo tem o objetivo de avaliar a influência aguda do uso de diferentes PB na capacidade aeróbica, potência muscular e metabólica em atleta. 


\section{METODOLOGIA}

\subsection{Amostra:}

Foi selecionado um jogador de basquetebol profissional com 26 anos, massa corporal de 75 $\mathrm{kg}$, estatura de $190 \mathrm{~cm}$ e classificado como respirador nasal. O participante foi notificado sobre os procedimentos de investigação, benefícios e riscos antes de fornecer o seu consentimento por escrito. O protocolo $(2.731 .493$ / 2018) foi aprovado pelo Comitê de Ética em Pesquisa dos Institutos Superiores de Ensino do CENSA (ISECENSA), Campos dos Goytacazes, Brasil.

\subsection{Confecção do Protetor Bucal:}

A confecção do protetor bucal tipo III foi realizada uma moldagem da arcada superior do atleta com alginato (Hydrogum 5 - Zhermack) e depois foi vazado esse molde com gesso pedra (Herodent Tipo III - Coltene) nas proporções adequadas para a obtenção do modelo em gesso da arcada dentária. De acordo com Queiróz (2013), foi utilizado uma placa de polivinil etileno acetato (EVA) semi-rígida de $3 \mathrm{~mm}$ de espessura (Placa para Moldeira Soft - Bio-Art) que foi colocada em uma plastificadora a vácuo (PlastVac P7 - Bio-Art), juntamente com o modelo de gesso para confecção do protetor bucal tipo III (figura 1).

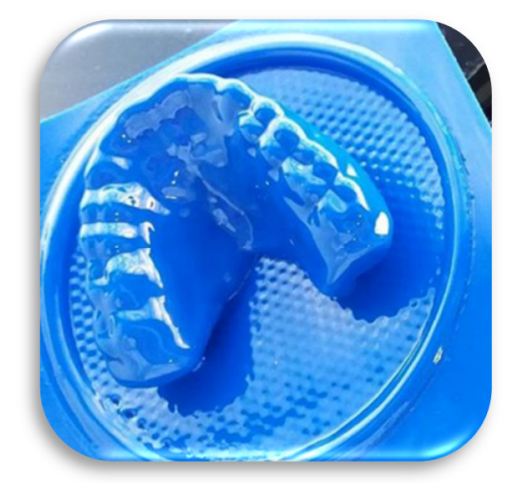

Figura 1. Protetor bucal (tipo III) confeccionado pelo Cirurgião Dentista

\subsection{Procedimento Experimental:}

Foi realizado um estudo randomizado cruzado: sem protetor bucal (Grupo Controle), protetor bucal universal (PBU) e protetor bucal personalizado (PBP). O atleta ao chegar ao ginásio poliesportivo foi coletado uma amostra sanguínea da polpa dos dedos e logo após realizou um aquecimento muscular (10min). O atleta realizou 2 baterias de teste constituído por 30 saltos verticais. O protocolo de Saltos Verticais foi realizado antes (AN) e imediatamente após (IA) a execução do teste da capacidade aeróbica. O protocolo de Saltos Verticais foi realizado em uma plataforma de saltos verticais (Cefise®, Brasil). Foi utilizada a variável potência muscular e realizadas as análises utilizando o tercil dos 30 saltos $\left(1^{\circ}\right.$ tercil: 10 Saltos; $2^{\circ}$ tercil: 10 Saltos; $3^{\circ}$ tercil: 10 Saltos). O teste da capacidade aeróbica utilizado seguiu o protocolo YO-YO Intermittent 
Recovery no nível 1. O protocolo consistiu em o atleta ir e voltar num trecho de 20 metros demarcados por cones, seguindo o ritmo de corrida determinado por uma sinalização sonora pelo software Beat Test Trainning (Cefise ${ }^{\circledR}$, Brasil). O teste teve o início com uma velocidade aproximada de $10.0 \mathrm{~km} / \mathrm{h}$ com pausas de $10 \mathrm{seg}$. a cada $40 \mathrm{~m}(2 \mathrm{x} \mathrm{20m})$. A velocidade foi incrementada em aproximadamente $0.5 \mathrm{~km} / \mathrm{h}$ a cada 1 minuto. $\mathrm{O}$ atleta foi induzido a completar a maior distância possível dentro do ritmo determinado pelos sinais sonoros. Para esse teste foi utilizado à distância máxima percorrida para as análises da capacidade aeróbica. $\mathrm{O}$ teste foi finalizado quando o atleta não conseguiu manter o ritmo dos sinais sonoros por duas tentativas. Imediatamente após a execução dos dois protocolos foi coletado mais uma amostra sanguínea. Os resultados foram obtidos a partir de um analisador portátil de lactato (Accutrend Plus, Roche ${ }^{\circledR}$, Alemanha). Os ensaios experimentais foram separados por $48 \mathrm{~h}$ (figura 2).

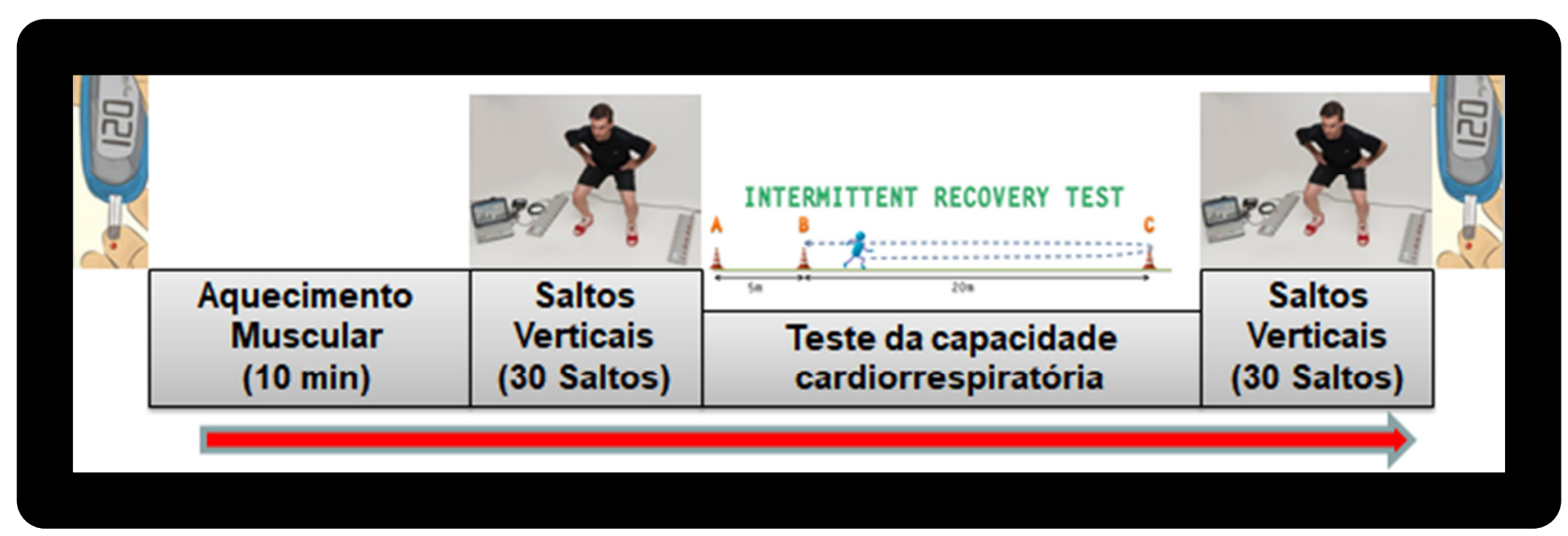

Figura 2: Desenho Experimental

\section{RESULTADOS E DISCUSSÃO}

Conforme a figura 3 a manutenção da potência muscular gerada pela plataforma de saltos verticais, seguiu o mesmo comportamento de redução entre os momentos antes e imediatamente após a realização do teste da capacidade aeróbica pelo atleta nos ensaios experimentais (Controle, PBU e PBP). No estudo de Drum et al., (2016) avaliando a manutenção da potência muscular em jogadores de futebol como um dos componentes da performance, não observou alterações significativas utilizando PB (personalizado e não personalizado) comparado ao controle (sem PB). Apesar desse estudo (2006) não tenha revelado o mecanismo específico para tais resultados observados, acredita-se que os benefícios na manutenção da potência muscular utilizando PB personalizados, podem estar relacionados à preensão natural dos dentes, que possivelmente reposicionaria a mandíbula durante o desempenho de um movimento explosivo (Salto Vertical), conferindo equilíbrio e coordenação durante a execução dos movimentos (Dunn-Lewis et al., 2012). Baseado nesse mecanismo e em estudos anteriores, Ebben et al. (2008) e Churei (2003) observaram benefícios na manutenção da taxa de potência e força muscular em testes específicos em atletas. 


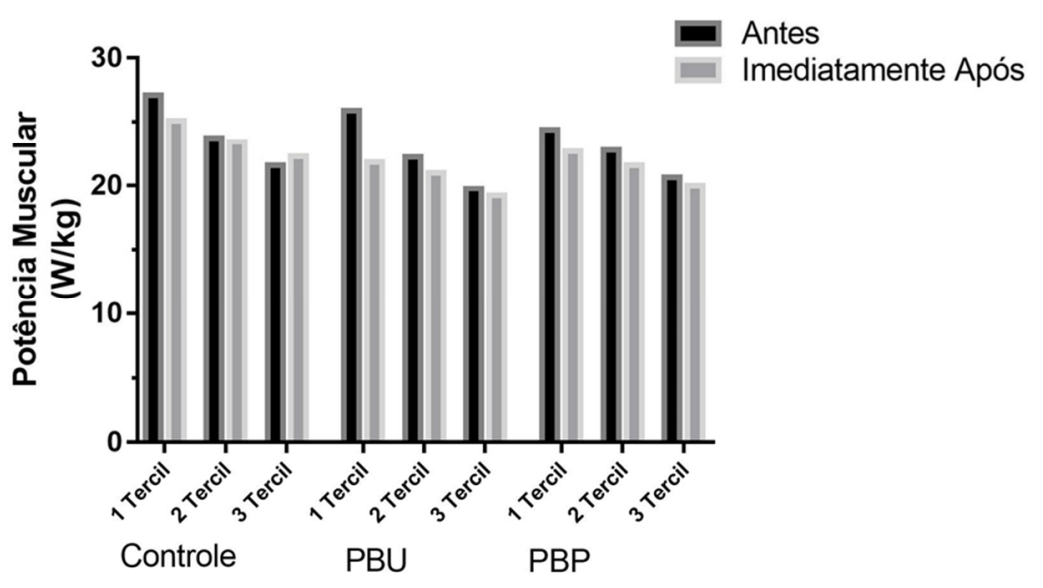

Figura 3: Análise da potência muscular antes e imediatamente após a realização do teste da capacidade aeróbica separados por tercil ( $1^{\circ}$ tercil: 10 Saltos; $2^{\circ}$ tercil: 10 Saltos; $3^{\circ}$ tercil: 10 Saltos) nos 3 ensaios experimentais. Controle - sem protetor bucal; PBU - protetor bucal universal; PBP protetor bucal personalizado.

É observado na figura 4 que as distâncias percorridas máximas atingidas foram de 2120 metros (PBP), 1530 metros (Controle) e 1255 metros (PBU). Diante desses resultados relacionados aos testes que envolvam o componente de endurance, autores como Gebauer et al. (2011) relatam que o uso de protetores não personalizados causa uma redução na patência das vias aéreas.

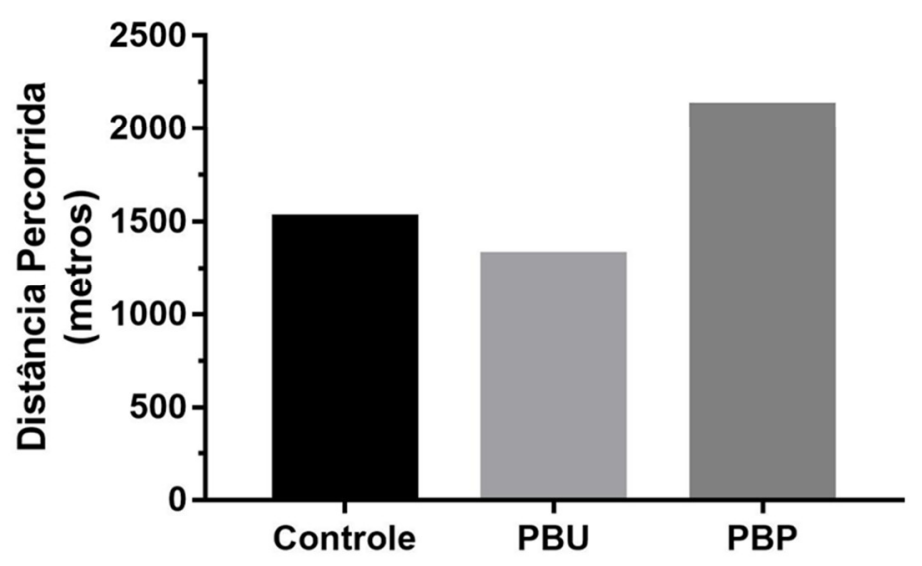

Figura 4: Análise da distância máxima percorrida no YO-YO Intermittent Recovery nos 3 ensaios experimentais. Controle - sem protetor bucal; PBU - protetor bucal universal; PBP - protetor bucal personalizado.

Especula-se que esse mecanismo pode ter influenciado na redução da distância percorrida máxima pelo atleta utilizando o PBU comparado ao controle, que, por conseguinte poderia ter afetado a concentração de lactado sanguíneo (figura 5 - Controle Antes: $4.1 \mathrm{mmol} / \mathrm{L}$, Imediatamente após: $16.9 \mathrm{mmol} / \mathrm{L} \Delta 12.8 \mathrm{mmol} / \mathrm{L}$; PBU Antes: $4.8 \mathrm{mmol} / \mathrm{L}$, Imediatamente após: $8.8 \mathrm{mmol} / \mathrm{L} \Delta$ $4.0 \mathrm{mmol} / \mathrm{L}$ ). Além disso, há evidências que o uso do PBP pode ajudar a manter a mandíbula estabilizada e, induzir uma melhora na função aeróbica por deixar as vias aéreas mais livres e assim 
gerando uma quantidade suplementar de $\mathrm{O}_{2}$ (dados não analisados diretamente pelo presente estudo) (Kerr, 1986; Queiróz et al. 2013). Acredita-se que esse benefício pode ter configurado positivamente em atingir uma distância maior percorrida pelo atleta com o uso do PBP, que consequentemente pode ter induzindo um incremento na concentração de lactato sanguíneo (PBP Antes: $8.4 \mathrm{mmol} / \mathrm{L}$, Imediatamente após: $20.1 \mathrm{mmol} / \mathrm{L} \Delta 11.7 \mathrm{mmol} / \mathrm{L}$ ) (figura 4 e figura 5). Esse aumento na concentração de lactato pode ser explicado pela maior solicitação da via glicogenolítica e/ou glicolítica. Sabe-se que aumento da hidrólise de ATPs muscular durante o esforço gradual, aumenta a concentração de $\mathrm{H}^{+}$livres levando a acidificação do meio (Cairns, 2016). Portanto, quanto mais ácido ficar o meio intra-celular e quanto mais lactato for produzido, mais $\mathrm{NAD}^{+}$ (coenzima na forma oxidada produzida entre a reação do piruvato e lactato) tende a se associar aos prótons $\left(\mathrm{H}^{+}\right)$. Esta reação tende a contribuir na regulação do $\mathrm{pH}$ celular removendo prótons do meio (Ishii \& Nishida, 2013). Acredita-se que o aumento na concentração sanguínea de lactato do atleta de basquetebol (utilizando PBP) funcionou como um "tampão auxiliar", o que pode ter proporcionado a melhora da performance.

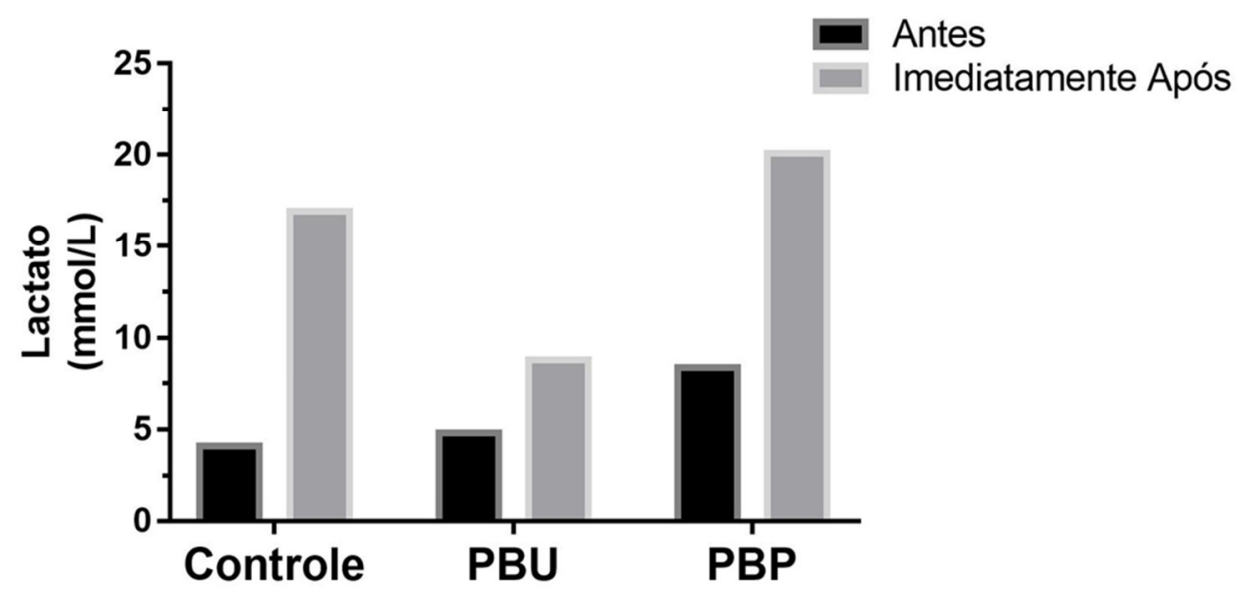

Figura 5: Análise da concentração de lactato sanguíneo antes e imediatamente após a realização dos testes nos 3 ensaios experimentais. Controle - sem protetor bucal; PBU - protetor bucal universal; PBP - protetor bucal personalizado.

Diante dos resultados observados em relação ao tratamento dos dados de forma descritiva e considerando um atleta pesquisado, os autores alertam sobre a extrapolação de determinadas inferências sobre o assunto.

\section{CONSIDERAÇÕES FINAIS}

Em conclusão, o presente estudo evidencia de forma descritiva que o uso do protetor bucal personalizado pode impactar no aumento da distância máxima percorrida pelo atleta, que consequentemente pode ter elevado à concentração de lactato sanguíneo. Já a manutenção da potência muscular foi similar entre os ensaios experimentais. Estes achados fornecem evidências adicionais para treinadores que defendem o uso de PB personalizados, não só para proteção

Persp. online: biol. \& saúde, Campos dos Goytacazes, 28 (8), 15-23, 2018

seer.perspectivasonline.com.br 
orofacial, mas também na indução de modificações positivas em determinados parâmetros específicos como a capacidade aeróbica e metabólica avaliados no atleta de basquetebol classificado como respirador nasal.

\section{REFERÊNCIAS}

CAIRNS, S.P. Lactic acid and exercise performance: culprit or friend? Sports Medicine, v.36; n.4, p.279-291, 2006.

CASTALDI, C.R. Athletic mouthguards: History and present status. The Sport Digest, v.10, p.1-2, 1988.

CASTALDI, C.R. Sports-related oral and facial injuries in the young athlete: A new challenge for the pediatric dentist. Journal of Pediatric Dentistry, v.08, n.4, p.311-316, 1986.

CHUREI, H. Relation between teeth clenching and grip force production characteristics. Kokubyo Gakkai Zasshi, v.70, n.2, p.82-88, 2003.

DRUM, S.N.; SWISHER, A.M.; BUCHANAN, C.A.; DONATH, L. Effects of a custom bitealigning mouthguard on performance in college football players. The Journal of Strength \& Conditioning Research, v.30, n.5, p.1409-1415, 2016.

DUNN-LEWIS, C. et al. The effects of a customized over-the-counter mouth guard on neuromuscular force and power production in trained men and women. The Journal of Strength \& Conditioning Research, v.26, n.4, p.1085-1093, 2012.

EBBEN, W.P.; FLANAGAN, E.P.; JENSEN, R.L. Jaw clenching results in concurrent activation potentiation during the countermovement jump. The Journal of Strength \& Conditioning Research, v.22, n.6, p.1850-1854, 2008.

FRONTERA, R.R: Traumatismo orofacial durante a prática de basquetebol e nível de informação sobre trauma e protetor bucal. Dissertação (Mestrado em Odontologia). Universidade São Leopoldo Mandic. Campinas, 2008.

GEBAUER, D.P.; WILLIAMSON, R.A.; WALLMAN, K.E.; DAWSON, B.T. The effect of mouthguard design on respiratory function in athletes. Clinical Journal of Sport Medicine, v.21, n.2, p.95-100, 2011.

ISHII, H; NISHIDA, Y. Effect of Lactate Accumulation during Exercise-induced Muscle Fatigue on the Sensorimotor Cortex. Journal of Physical Therapy Science, v.25, n.12, p.16371642, 2013.

KAUFMAN, R.S. Case reports of TMJ repositioning to improve scoliosis and the performance by athletes. NY State Dental Journal, v.46, n.4, p.206-209, 1980.

KERR, I. L. Mouth guards for the prevention of injuries in contact sports. Sports Medicine, v.3, n.6, p.415-427, 1986.

N.Y.S.S.F. National Youth Sports Safety Foundation; Sports Dentistry facts: facts from the National Youth Sports Foundation for Safety. Disponível em: www.sportsdentistry/facts, acesso em: 04/12/18.

PIERO, M. et al. Influence of a custom-made maxillary mouthguard on gas exchange parameters during incremental exercise in amateur road cyclists. The Journal of Strength \& Conditioning Research, v.29, n.3, p.672-677, 2015. 
QUEIRÓZ, A.F.V.R.; DE BRITO J.R.R.B.; RAMACCIATO, J.C.; MOTTA, R.H.L.; FLÓRIO, F.M. Influence of mouthguards on the physical performance of soccer players. Dental Traumatology, v.29, n.6, p.450-454, 2013.

SCHWARTZ, R.; NOVICH, M.M. The athlete's mouthpiece. The American Journal of Sports Medicine, v.8, n.5, p.357-359, 1980.

STEWART, G.B; SHIELDS, B.J; FIELDS, S; COMSTOCK, R.D; SMITH, G.A. Consumer products and activities associated with dental injuries to children treated in United States emergency departments 1990-2003. Dental Traumatology, v.25, n.04, p.399-405, 2009. 